Article

\title{
The Moon as a Climate-Quality Radiometric Calibration Reference
}

\author{
Thomas C. Stone ${ }^{1, *}$, Hugh Kieffer ${ }^{2}$, Constantine Lukashin ${ }^{3}$ and Kevin Turpie ${ }^{4}$ \\ U.S. Geological Survey, Flagstaff, AZ 86001, USA \\ Celestial Reasonings, Genoa, NV 89411, USA; hhkieffer@gmail.com \\ NASA Langley Research Center, Hampton, VA 23681, USA; constantine.lukashin-1@nasa.gov \\ 4 NASA Goddard Space Flight Center, Greenbelt, MD 20771, USA; kevin.r.turpie@nasa.gov \\ * Correspondence: tstone@usgs.gov; Tel.: +1-928-556-7381
}

Received: 23 April 2020; Accepted: 3 June 2020; Published: 5 June 2020

check for updates

\begin{abstract}
On-orbit calibration requirements for a space-based climate observing system include long-term sensor response stability and reliable inter-calibration of multiple sensors, both contemporaneous and in succession. The difficulties with achieving these for reflected solar wavelength instruments are well known. The Moon can be considered a diffuse reflector of sunlight, and its exceptional photometric stability has enabled development of a lunar radiometric reference, manifest as a model that is queried for the specific conditions of Moon observations. The lunar irradiance model developed by the Robotic Lunar Observatory (ROLO) project has adequate precision for sensor response temporal trending, but a climate-quality lunar reference will require at least an order of magnitude improvement in absolute accuracy. To redevelop the lunar calibration reference with sub-percent uncertainty and SI traceability requires collecting new, high-accuracy Moon characterization measurements. This paper describes specifications for such measurements, along with a conceptual framework for reconstructing the lunar reference using them. Three currently active NASA-sponsored projects have objectives to acquire measurements that can support a climate-quality lunar reference: air-LUSI, dedicated lunar spectral irradiance measurements from the NASA ER-2 high altitude aircraft; ARCSTONE, dedicated lunar spectral reflectance measurements from a small satellite; and Moon viewing opportunities by CLARREO Pathfinder from the International Space Station.
\end{abstract}

Keywords: lunar calibration; radiometric reference; spectral irradiance; stability; Moon

\section{Introduction}

To detect the subtle signatures of climate change requires continuous observations of Earth environmental variables over decadal time periods. But satellite systems are not expected to remain operational for such time spans, and the climate task requires separating actual changes in the terrestrial observables from any drift in the instruments themselves. Therefore, the sensors of a space-based climate observing system must be re-calibrated regularly in flight to evaluate drift effects with high precision, and successions of instruments must be cross-calibrated with high accuracy to assure inter-consistency across the multiple instruments and platforms that are tasked with making long-term observations.

There are well-known difficulties in realizing on-orbit calibrations for radiometer instruments in the reflected solar wavelength range, $\sim 350 \mathrm{~nm}$ to $2500 \mathrm{~nm}$. The discussion presented here pertains exclusively to that wavelength regime. On-board calibration systems using solar diffusers and/or reference ground targets have inherent limits on their achievable accuracy, primarily due to degradation of the diffuser material in the space environment and corrections for atmospheric transmission in the case of vicarious calibration using ground targets. 
The capability exists to utilize the light from the Moon as a reference standard for radiometric calibration of reflected solar wavelength sensors. The Moon can be considered an ultra-stable solar diffuser, and a spatially extended object with a reflectance range similar to clear land scenes on Earth. The apparent lunar brightness sensed by an observer (whether human or a photodetector) is governed by the geometry of the observation, primarily the Sun-Moon-observer angle or phase angle, coupled with the angle-dependent reflectance properties of the lunar surface. Since the positions of the Sun, Moon and an observer (and thus the observation geometry) typically can be known with high accuracy, the Moon's brightness can be predicted given a photometric specification for the lunar surface reflectance and the solar irradiance. This prediction capability is the foundation for building a radiometric reference from moonlight.

From the vantage point of an observatory on Earth or in low Earth orbit, the lunar brightness varies in a smooth and continuous manner. Consequently, to accommodate the unique illumination and viewing conditions of arbitrary Moon views requires the predictive capability to be continuous in the observation geometry parameter space. This is accomplished by employing an analytic model as the reference, where the model can be queried for the specific conditions of Moon observations.

The validity of a lunar model derives from the temporally invariant nature of the lunar surface. Having no atmosphere, the Moon has been subject to eons of space weathering that has pulverized the lunar regolith, stabilizing the reflectance and nearly completely erasing any spectral structure of the surface constituents. Studies of the Moon's photometric stability have concluded that it is invariant at a level less than one part in $10^{8}$ per year [1]. Consequently, a model-based lunar reference potentially can have exceptional absolute accuracy and relative precision. Such a model is then valid for comparisons of lunar observations made at any time, including the past.

Several important calibration capabilities are enabled by a lunar radiometric reference as a direct consequence of the Moon's inherent stability. Currently, the most common application for lunar calibration is tracking sensor response changes over time, where drifts can be characterized with sub-percent precision given a sufficiently long time series of Moon observations. This has been demonstrated for the Sea-viewingWide Field-of-view Sensor (SeaWiFS) ocean color instrument [2]. Inter-calibration can be achieved with high precision using the Moon as a common target without the need for simultaneous observations; however, a model must be employed to predict the differences between the sensors' lunar measurements, since each view of the Moon has a unique observation geometry. The common Moon target also can be used to bridge a gap in an otherwise contiguous record of Earth observations, but the instruments both before and after the gap must have acquired lunar measurements, and the accuracy of the bridge calibration depends critically on the accuracy of both the instrument measurements and the lunar reference. The capability for cross-calibration can be extended to the transfer of pre-launch calibration to on-orbit operations.

Lunar calibration also can be applied for back-calibration of archived image data that include Moon views, such as from geostationary meteorological satellite imagers. The Moon is regularly captured in the margins and corners of a scanning imager's rectangular field of regard. Analysis of lunar radiometric measurements from these Moon images offers the potential to develop consistent calibrations spanning decades, and across multiple satellites covering different parts of the globe.

At present, the most commonly used lunar models cannot be verified for absolute accuracy with better than about $5 \%$ uncertainty. But the invariant nature of the lunar surface means that absolute radiometry of the Moon and specifying its photometric behavior with illumination and view geometry can be done with extremely high accuracy and relative precision. Improvements of at least an order of magnitude over current uncertainties are possible, and these are needed to fully exploit the possibilities enabled by absolute lunar calibration.

This paper presents the conceptual components of a new, high-accuracy lunar radiometric reference that can tap the full potential of the Moon as a calibration target. To set context, the current state of lunar model development and its known limitations are reviewed. The potential capabilities and requirements for a climate-quality lunar reference are given, along with specifications for new 
measurements needed to support its construction. Some examples are presented of recent active efforts to acquire high-accuracy lunar measurements. The final section provides a conceptual framework for methodologies to utilize these new measurements to refine the existing lunar reference and for advanced lunar modeling to redevelop the reference.

\section{Lunar Calibration System Development}

The ability to construct a calibration reference from moonlight follows from obtaining a detailed characterization of how the lunar brightness varies with time and the location of the observer, plus any spectral dependencies. The precision with which the reference can specify the Moon's brightness for an arbitrary observation geometry depends on the extensiveness of the characterization measurements.

The variations in albedo over the Moon's surface present a non-uniform radiance field to an observer. Directly comparing lunar images acquired by a sensor to a spatially resolved (radiance) reference must specify a particular region of the Moon to be used. Because the lunar surface is variegated at all spatial scales, precise registration with the same Modulation Transfer Function (MTF) would be required. Nonetheless, using a lunar radiance reference may be necessary for sensors with a small field of regard, which can make sampling the entire lunar disk impractical.

The difficulties with using a spatially resolved reference are mitigated by using the radiometric quantity of irradiance for lunar calibration, which involves spatially integrating Moon radiance images. This approach gains signal-to-noise from summing individual radiance measurements (image pixels), and simplifies the reference by treating the Moon's brightness as a scalar value for a given wavelength.

Using the lunar disk in its entirety, however, means the reference must explicitly account for the particular hemispheres that are illuminated and viewed, known as the lunar librations. Consequently, the characterization measurements must sufficiently cover the libration parameter space, access to which is constrained by the Moon's orbit. From a point fixed on the Earth, the lunar hemisphere that is observed changes with time in a manner such that the sub-observer point on the Moon's surface traces a pattern similar to a Lissajous figure. To sample all regions of the space defined by the sub-observer selenographic longitude and latitude requires three to five years, depending on when the sequence is started. Adding full coverage of phase variations increases the time required to sample the parameter space to 18.6 years. This circumstance historically has limited efforts to acquire lunar characterization measurements to only a few projects and institutions.

\subsection{Moon Observations at the U.S. Geological Survey}

The prototype lunar calibration system was developed by the Robotic Lunar Observatory (ROLO) project at the U.S. Geological Survey (USGS) Astrogeology Science Center in Flagstaff, Arizona, under sponsorship from the National Aeronautics and Space Administration (NASA) Earth Observing System (EOS) program. Observations acquired by the ROLO telescopes form the basis for the lunar irradiance model [3] discussed in the next section. The ROLO telescope facility was constructed in the 1990s, and operated every clear night between First Quarter and Last Quarter lunar phases from 1995 to 2003. Typically the Moon was observed between 3 and 15 times during a night, depending on the phase, collecting images in 32 spectral bands each time. Altogether more than 110,000 images of the Moon were acquired, along with more than $10^{6}$ star images to characterize atmospheric transmission and correct the Moon observations to exo-atmospheric. Figure 1 shows the geometric coverage of the ROLO lunar database from processing version 3.11, ca. 2003. 


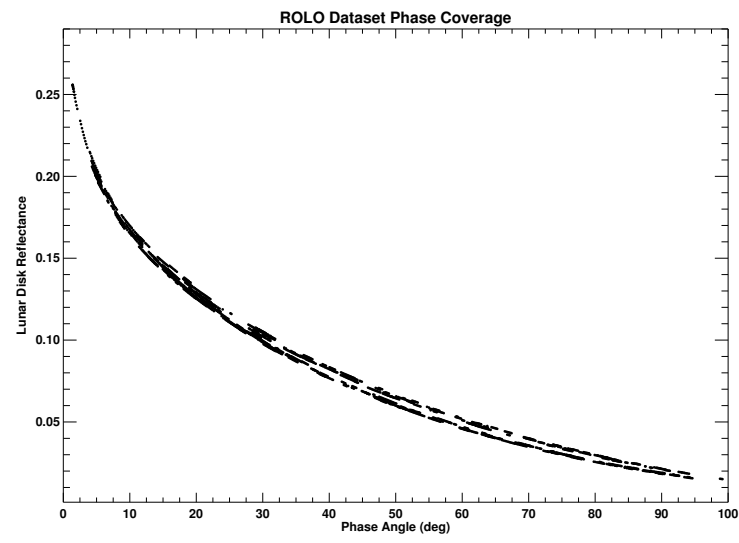

(a)

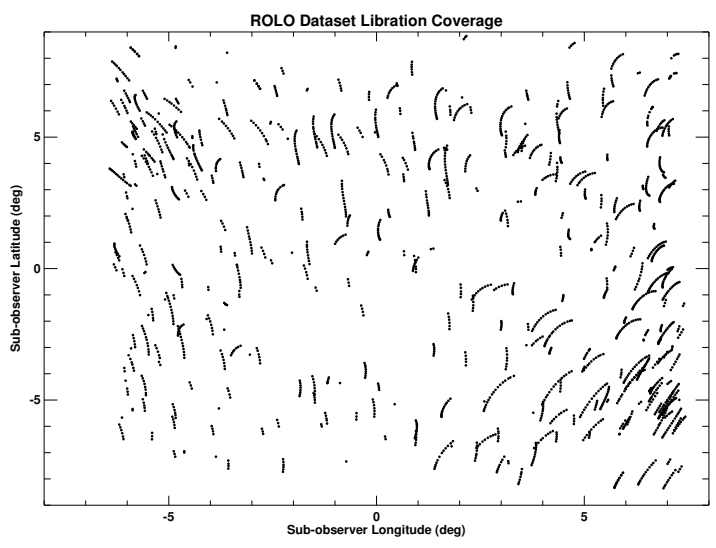

(b)

Figure 1. Geometric observation parameter coverages for the ROLO data that were used to construct the USGS lunar irradiance model. The dot symbols represent sequences of images acquired in all 32 ROLO bands; 1200 such sequences are represented here. (a) Lunar disk reflectance phase curve for the ROLO $555 \mathrm{~nm}$ band, showing phase angle coverage and the bimodal waxing/waning distribution.

(b) Lunar libration coverage, plotted as selenographic sub-observer longitude and latitude coordinates.

The twin ROLO telescopes each have $20 \mathrm{~cm}$ diameter primary mirrors, and are on-axis Ritchey-Crétien optical designs for virtually distortion-free imaging in the visible and near-infrared (VNIR) and shortwave infrared (SWIR) wavelength ranges. The VNIR camera is a $512 \times 512$ pixel charge-coupled device (CCD) array, sized to give a similar spatial resolution as the Advanced Spaceborne Thermal Emission and Reflection Radiometer (ASTER) instrument on the Terra satellite. It has 23 narrow-band filters from $350 \mathrm{~nm}$ to $945 \mathrm{~nm}$. The SWIR camera is a $256 \times 256$ pixel HgCdTe photoconductive array, with 9 filters from $945 \mathrm{~nm}$ to $2450 \mathrm{~nm}$, operating at liquid nitrogen temperature. On each observing night an autonomous data acquisition system [4] created a program of Moon and star observations, then executed instructions for automated centering on targets, setting filter wheel positions and camera integration times, and positioning a pair of movable neutral density filters in and out of the optical paths for Moon and star imaging, respectively.

Star observations were collected all night (weather permitting), regardless of whether the Moon was above the horizon. The star images are spatially integrated to irradiances (in instrument units, $\mathrm{DN} / \mathrm{sec}$ ) to obtain atmospheric extinction measurements, which are used in a multivariate fitting routine that performs Langley analysis on all star measurements in all 32 ROLO spectral bands simultaneously. The procedure is described in detail in [3]. The outcome of these nightly analyses is a parameterized expression that characterizes the optical transmission of the atmosphere above the observatory as a function of position in the sky and time. This expression is then applied for the conditions of the Moon observations to generate atmospheric corrections for these data.

The calibration coefficients used to convert ROLO Moon images to radiance were derived from observations of the star Vega ( $\alpha$ Lyr), comparing the ROLO instrument response to published absolute stellar fluxes [5,6]. The images of Vega were processed to stellar irradiances identically to the other ROLO star observations, for use in the atmospheric characterization routine discussed above. Atmospheric transmission corrections were derived and applied to the Vega observations. Absolute photon fluxes for Vega in each ROLO band were found by scaling the Kurucz stellar spectrum [7] to the absolute references [5,6] and convolving with the ROLO band spectral responses. Factors to quantify the neutral density filter attenuation were derived empirically from periodic special measurements of Vega with the filters in and out of the optical paths. Applying this point-source calibration to the entire focal plane array requires that the array equalization (flatfielding) is considered reliable. 


\subsection{ROLO Lunar Irradiance Model}

The ROLO model for lunar spectral irradiance currently is widely used as a radiometric reference, and has been replicated many times for various purposes, e.g., [8]. Details of its development can be found in [3]. Although ROLO model outputs are lunar spectral irradiance, model development and operation are done in terms of lunar disk-equivalent reflectance. Operating in reflectance space avoids the complexity of the solar spectral structure and takes advantage of the smoothness of the lunar reflectance spectrum, which has only broad, shallow absorption features.

To prepare the ROLO dataset for constructing the reflectance model, measurements of lunar irradiance $E_{\text {meas }}$ are derived from spatial integration of the Moon radiance images:

$$
E_{\text {meas }}=\Omega_{\mathrm{p}} \sum_{i=1}^{N_{\mathrm{p}}} L_{i}
$$

where $\Omega_{\mathrm{p}}$ is the solid angle of the image pixels, $L_{i}$ is the net radiance of pixel $i$, and the sum covers all pixels on the Moon disk $N_{\mathrm{p}}$, including the unilluminated portion. In practice, this process accounts for image edge spread effects and moonlight scattering by the atmosphere. Because the ROLO instruments are framing cameras, there is no oversampling. The measured irradiances are adjusted to standardized distances of $1 \mathrm{AU}$ for the Sun-Moon distance and 384,400 km (the mean lunar orbit radius) for the Moon-observer distance:

$$
E_{\text {meas }}^{\prime}=E_{\text {meas }}\left(\frac{d_{\text {Sun-Moon }}}{1 \mathrm{AU}}\right)^{2}\left(\frac{d_{\text {Moon }- \text { ROLO }}}{384,400 \mathrm{~km}}\right)^{2}
$$

The distances $d_{\text {Sun-Moon }}$ and $d_{\text {Moon-ROLO }}$ are computed for the acquisition times of the images using high-precision ephemerides for the Sun and Moon. The irradiance measurements are then converted to disk reflectance $A_{\text {meas }}$ utilizing the solar spectral irradiance $E_{\text {Sun }}$ in each ROLO band $k$ :

$$
A_{\text {meas }, k}=\frac{E_{\text {meas }}^{\prime}}{\Omega_{\text {Moon }} E_{\text {Sun }, k} / \pi}
$$

where $\Omega_{\text {Moon }}=6.41775 \times 10^{-5} \mathrm{sr}$ is the solid angle of the Moon at the standard Moon-observer distance $(384,400 \mathrm{~km})$.

At the time of the ROLO dataset processing that was used for lunar model development (version 3.11, early 2000s), the commonly used solar irradiance spectrum of Thuillier [9] was not yet available. Therefore, $E_{\text {Sun }, k}$ values were derived using the solar spectrum published in 1985 by the Physikalisch-Meteorologisches Observatorium Davos (PMOD) (Wehrli, [10]).

Formulating the ROLO model took an empirical approach, with the primary driver being minimizing the residuals of fitting the reflectance dataset. The model form specifies the lunar disk-equivalent reflectance $A_{k}$ at each ROLO band $k$ corresponding to inputs of the geometric variables of phase angle $g$ and the lunar librations, expressed as the selenographic sub-observer longitude $\phi$ and latitude $\theta$ and sub-solar longitude $\Phi$ :

$$
\begin{array}{r}
\ln A_{k}=\sum_{i=0}^{3} a_{i k} g^{i}+\sum_{j=1}^{3} b_{j k} \Phi^{2 j-1}+c_{1} \phi+c_{2} \theta+c_{3} \Phi \phi+c_{4} \Phi \theta \\
+d_{1 k} e^{-g / p_{1}}+d_{2 k} e^{-g / p_{2}}+d_{3 k} \cos \left(\left(g-p_{3}\right) / p_{4}\right)
\end{array}
$$

This expression is a continuous function of the time-associated phase and libration parameters, and accounts for the waxing/waning difference and the opposition surge. The 328 coefficients $a, b, c, d$ and $p$ were found by fitting the ROLO measured reflectances (Equation (3)) independently for each of the 32 ROLO bands. Their values are listed in [3], and are available in electronic form for replicating the model by contacting the corresponding author of this paper. 
Operating the lunar model to generate radiometric reference values involves processing details of instruments' Moon observations and their sensor spectral response characteristics. Typically instrument teams provide the times and instrument locations for their Moon observations, and the ROLO team converts these to the phase and libration variables $(g, \phi, \theta, \Phi)$ using ephemerides for the Moon and the Sun. Computing Equation (4) produces 32 disk reflectance values $A_{k}$ corresponding to the ROLO bands. The resulting spectrum exhibits irregular structure that is not representative of the Moon's actual reflectance spectrum, which is smooth, with only broad, shallow absorption features. Therefore, the outputs of Equation (4) are spectrally smoothed by fitting a representative lunar reflectance spectrum that was developed from laboratory spectra of returned Apollo samples [11] and is considered suitable to capture the spectral content of the lunar disk as a whole. Figure 2 shows an example of this process, where the necessity for spectral smoothing is clearly seen. This fitting process employs a least absolute deviation method of linear regression to give a best fit to all $32 A_{k}$ results, while also being constrained by the fixed spectral shape of the representative reflectance spectrum. The relatively large deviations seen at the two longest wavelength bands originate with the ROLO observational dataset, and show a need for data processing refinements.

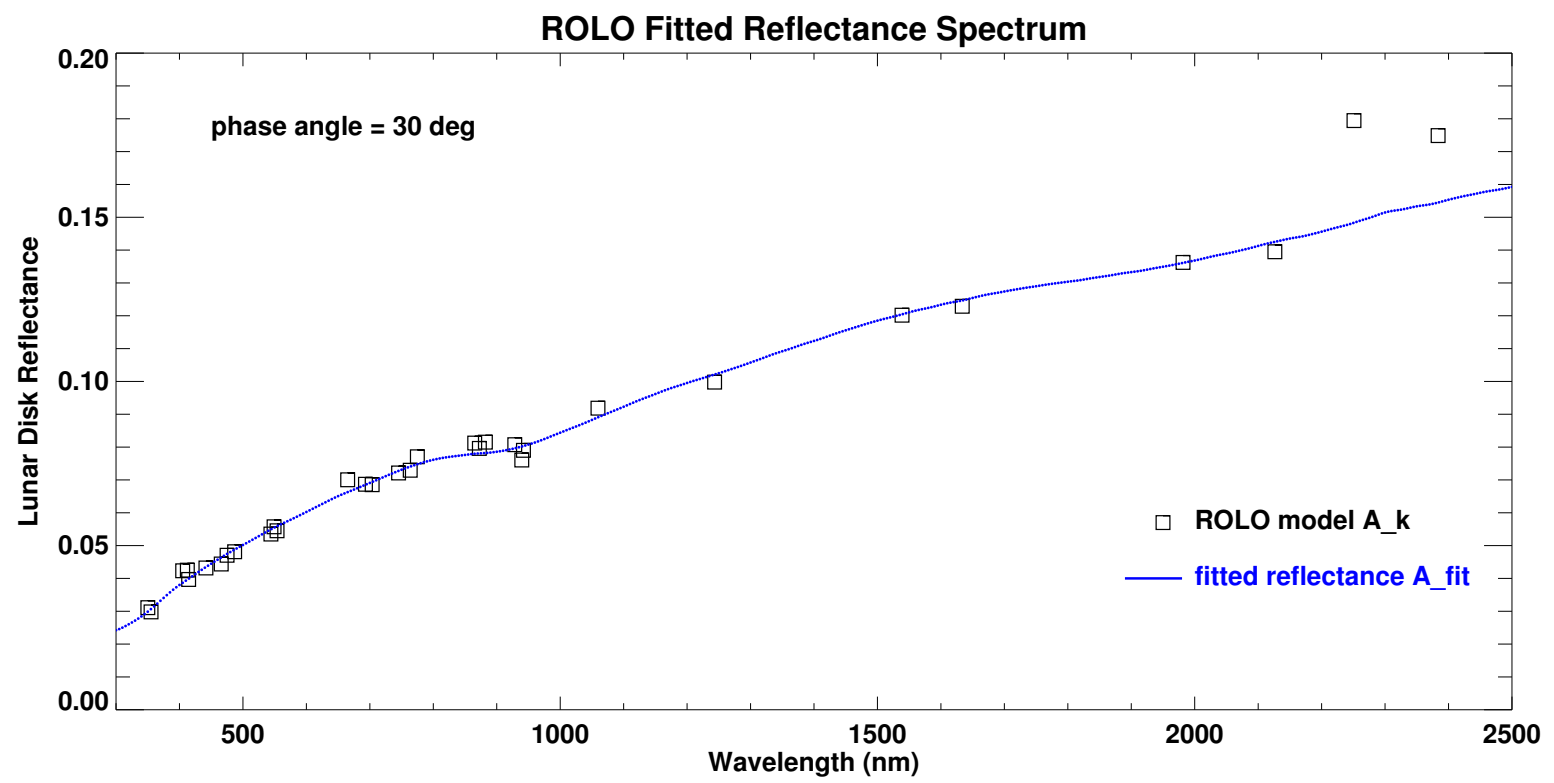

Figure 2. Example of fitting the representative lunar reflectance spectrum (blue line) to the model-generated disk reflectance outputs of Equation (4) (square symbols).

The reference lunar irradiance specific to an instrument's spectral bands $E_{\text {ref }}$ is found by interpolating the fitted disk reflectance spectrum and convolving with the sensor's spectral response and the solar spectral irradiance:

$$
E_{\text {ref }}=\frac{\Omega_{\text {Moon }}}{\pi} \frac{\int A_{\text {fit }}(\lambda) E_{\text {Sun }}(\lambda) S(\lambda) d \lambda}{\int S(\lambda) d \lambda}
$$

where $\Omega_{\text {Moon }}$ is the solid angle of the Moon as above, $A_{\text {fit }}(\lambda)$ is the fitted lunar reflectance spectrum, $E_{\text {Sun }}(\lambda)$ is the solar spectral irradiance, and $S(\lambda)$ is the sensor band spectral response. Since the same fitted spectrum $A_{\text {fit }}(\lambda)$ is used for all sensor bands, the results are coupled across the bands.

The $E_{\text {ref }}$ values are adjusted to the actual Sun-Moon and Moon-sensor distances of the observations using the inverse of Equation (2). The final results are the reference lunar spectral irradiances corresponding to the time and location of the instrument's Moon observations, for the sensor's spectral bands. 


\section{Practical Application of the Lunar Calibration System}

\subsection{Lunar Irradiance Measurements by Instruments}

For instruments to measure lunar irradiance for comparison against the model-based reference, the complete Moon disk must be sampled. Measurements from images, such as those produced by line scanning sensors, are derived from summing radiance pixels:

$$
E_{\text {meas }}=\frac{\Omega_{\mathrm{p}}}{\eta} \sum_{i=1}^{N_{\mathrm{p}}} L_{i}
$$

where $\Omega_{\mathrm{p}}$ is the solid angle of acceptance for a pixel, $\eta$ is the oversampling factor, and $L_{i}$ is the net radiance of pixel $i$ on the Moon disk. Since each of these parameters factor linearly into the measurement equation, careful evaluation of each is critical to the accuracy of the measured irradiances, and incomplete accounting of them can lead to significant measurement biases.

Quantifying the solid angle $\Omega_{\mathrm{p}}$ requires evaluating the effective spatial response of the detectors that form the image pixels. Substituting ground sampling distances (an often-used shortcut) presumes the spatial response functions are perfect step functions. Deviations from this ideal form will cause systematic measurement errors for any irradiance target, including the Moon.

Lunar images from line-scanning sensors are produced by concatenating series of frames (scan lines) acquired while slewing the sensor view field across the Moon, typically with a frame acquisition rate that results in oversampling. Processing these images into lunar irradiances requires precise knowledge of the actual oversampling of the Moon disk, which is different from the ratio of sampling to slew rates. Determining the oversampling must account for drift in the position of the Moon target relative to the sensor line of sight, and rotation of the Moon's orientation relative to the scan direction, both of which can result from spacecraft orbital motion during scanning.

The pixel radiances $L_{i}$ are net radiances, above background. The space-viewing regions surrounding the Moon disk can provide a high quality reference for evaluating the zero-radiance background level of lunar images.

The most reliable lunar irradiance measurements are obtained when the Moon is observed using the same optical and sensor electronics configurations as for normal operations, i.e., the normal nadir viewing configuration. For instruments in low Earth orbit, this requires an attitude maneuver to look above the horizon, which may alter the thermal state of the instrument. If a space-view port can be used to view the Moon, periodic space-view observations closely preceding or following such direct-view observations can help quantify any biases or trends that may result from these maneuvers.

Moon observations have been included in the flight calibration plans for many Earth observing satellite missions, and substantial lunar calibration records have been produced to date, including a nearly 20-year series acquired by Terra MODIS [12]. The lunar measurements acquired over the lifetime of SeaWiFS led to stabilizing its sensor response with sub-percent precision [2]. For relative calibrations such as the example of temporal response trending, the series of instrument-to-reference comparisons typically get normalized to a point early in the mission $[2,13,14]$. But the absolute irradiances measured by instruments often differ substantially from the corresponding ROLO reference values, by amounts which can exceed the estimated uncertainties of both the instrument radiometric calibrations and the ROLO model. This suggests a need for closer examination of the instruments' irradiance measurements for potential sources of error such as the issues described above. But it also demands examination of the ROLO model construction for a potential origin; this is discussed next.

\subsection{Uncertainties in the ROLO Reference}

The irradiance results generated by the ROLO model via the processes described in Section 2.2 assume the absolute scale of the disk reflectance expression, Equation (4), which in turn reflects 
the radiometric calibration of the ROLO lunar image dataset and its correction for atmospheric transmission. These are the two largest sources of uncertainty in the ROLO lunar reference.

During ROLO telescope operations, the automated observation acquisition program selected 10 to 15 different stars from a list of about 200 non-variable star targets based on their positions in the sky and spanning a range of stellar colors. Each star was imaged in all 32 ROLO bands up to 12 times through the night. The normal setting for maximum viewing zenith angle was $60^{\circ}$, limiting the observation to an airmass range of 1 to 2 . After reducing the images to stellar irradiances, the atmospheric analysis routine fits the entire ensemble of star measurements simultaneously to quantify the radiative transmission over the extent of the sky covered by the observations. This routine keeps an accounting of the combined uncertainty of both the irradiance measurements and the Langley fits. The results vary depending on the ensemble of stars targeted and the night's viewing conditions (astronomical "seeing"), and differ among the ROLO bands. Under the best observing conditions, the calculated uncertainty in the atmospheric characterization approaches about $3 \%(\mathrm{k}=1)$.

The absolute stellar flux references for Vega that are used to define the ROLO radiometric scale have reported uncertainties of $1.5 \%$ at $500 \mathrm{~nm}$ [5] and 2-3\% over the SWIR range [6]. The ROLO calibration observations of Vega also are subject to the same uncertainty in the atmospheric transmission correction described above, leading to an estimated 3-4\% uncertainty in the ROLO radiometric calibration.

The ROLO lunar irradiance measurements used as the basis for modeling have additional uncertainties tied to the spatial integration of the Moon images and evaluation of the neutral density filter transmission. These factors are band-dependent, and combined give uncertainty estimates of $5-8 \%$ for the ROLO lunar measurement database. Analysis of the residuals from fitting the ROLO data to the disk reflectance model (Equation (4)) suggests that the model representation of the geometric irradiance variations has a relative uncertainty $\sim 1 \%$. However, the absolute accuracy of the ROLO lunar reference cannot be verified to better than about $5 \%$ uncertainty.

\section{Requirements for a Climate-Quality Lunar Reference}

The differences seen in comparisons of lunar irradiance measurements made by sensors against ROLO model results need to be better understood to exploit the full potential of the Moon as a radiometric reference, such as absolute lunar calibration and inter-calibration. Uncertainties in lunar irradiance measurements from images result from all the contributing factors described in Section 3.1 above, corresponding to the terms in Equation (6). An instrument's radiometric calibration influences only the pixel radiance values $L_{i}$. Uncertainty in ROLO lunar reference reflects a limitation of the current model only, whereas the Moon's brightness potentially can be known to the accuracy limits of radiometry conducted outside the controlled environment of a laboratory. Because lunar observations are by nature field measurements, excess uncertainties can be expected, which ultimately may impose a limit on the accuracy achievable for characterizing the Moon and developing a lunar reference, although this threshold can be expected to drop as measurement technologies advance.

For lunar calibration to meet the demanding requirements of climate applications, at least an order of magnitude improvement in accuracy over the current lunar reference is needed. Efforts toward refinement of the ROLO system continue, but the limitations of the ROLO dataset pertaining to its atmospheric correction reveal a critical need for new absolute lunar measurements, including some observations acquired from above the atmosphere. A number of specifications for new characteristic measurements of the Moon have been identified; overviews are given here.

\subsection{Geometric Variation Specification}

The usefulness of a model-based lunar reference derives from its ability to predict the Moon's brightness for any given time and location on the Earth's surface or along a spacecraft's orbit track. Of the geometric parameters governing lunar irradiance, the inverse-square distance dependencies can be determined accurately by computing a high-precision ephemeris. The precision with which a 
model can predict the variations in lunar brightness with phase angle and librations depends on the density and extent of coverage of these parameters in the dataset(s) used as the basis for modeling.

Collecting lunar characterization measurements from a ground-based observatory permits regular instrument maintenance and calibration, enabling years-long campaigns that can sample a wide range of phase angles and librations despite anticipated interruptions caused by weather. But ground-based measurements are restricted to phase angles less than about $90^{\circ}$ (First Quarter to Last Quarter) by the requirement to observe the Moon only at night. The Moon's orbit governs the phase angles and libration states available for viewing from the ground or from low Earth orbit, and the obliquity of the lunar orbit means a minimum 3 years of observations are required to sample all regions of the libration parameter space. The librations sampled during the $8+$ years of ROLO operations were shown in Figure 1.

Moon characterization measurements acquired from a space-based platform potentially can sample the Moon every orbit, with phase angle coverage extending to the limits of a Sun exclusion zone, although transferring pre-launch calibration to on-orbit operations remains an issue. Typical usage of lunar calibration for satellite Earth observing instruments is to track sensor performance over time, for which the lunar reference values are applied to offset the variations in irradiance arising from differences in the observed phase angles and librations. However, some lunar measurements acquired from space have exhibited self-consistency at a level such that they can be considered lunar characteristic measurements themselves. Such data potentially can provide useful supplements for lunar model development, although many Earth observing sensors sample only a limited range of phase angles.

\subsection{Absolute Accuracy and Traceability to Système International (SI) Units}

The accuracy achievable for Moon characterization measurements taken from the ground is limited by the uncertainty in evaluating the radiative transfer of moonlight through the atmosphere, where the magnitude of these corrections depends on wavelength, the altitude of the observatory (i.e., ambient atmospheric pressure), and variable meteorological conditions. Consequently, the extensive geometric coverage offered by a ground-based lunar measurement campaign needs to be supplemented by above-atmosphere measurements to reach tenths-percent uncertainty levels. Occasional measurements acquired from a high-altitude balloon or stratospheric aircraft platform can serve as tie points to anchor the absolute scale of a lunar model or a ground-based measurement set, while avoiding the complication of maintaining calibration of an instrument in orbit. This is an objective of the airborne Lunar Spectral Irradiance (air-LUSI) project, as described in a later section.

The capability for generating lunar radiometric reference values for the specific illumination and view geometries of Moon observations was developed from knowledge of the lunar surface reflectance, which is inherently stable [1] but exhibits strong dependencies on the angles of incidence and emission and the phase angle. The fundamental lunar reference standard is a reflectance standard, whereas the working radiometric reference must be derived from coupling this reflectance standard to the solar spectral irradiance (SSI) as explained in Section 2.2. Therefore, an SI-traceable lunar reference may be constructed from extensive characterization of the lunar surface reflectance with robust uncertainty evaluations, combined with the SSI obtained from SI-traceable measurements. To achieve the highest accuracy, time-resolved SSI must be utilized to account for solar variability, although this effect is relatively small for wavelengths longer than $350 \mathrm{~nm}$. Direct measurement of lunar disk-equivalent reflectance is the objective of the ARCSTONE mission, as described in a later section.

Another approach could be to measure the lunar spectral irradiance directly using an SI-traceable sensor in orbit. This is one objective for the Traceable Radiometry Underpinning Terrestrial- and Helio-Studies (TRUTHS) mission currently in development at the National Physical Laboratory of the United Kingdom. Lunar measurements acquired by TRUTHS will be calibrated to its on-board cryogenic electrical substitution radiometer. 


\subsection{Spectral Specification}

A spectrally resolved capability is an essential component of a viable lunar radiometric reference, to enable generating results for the different spectral bands of remote sensing instruments, with sufficient resolution to accommodate spectrometers. The smoothness and the broad, shallow absorption features of the lunar reflectance spectrum (cf. Figure 2) allow for continuous interpolation of reflectance model results, but new Moon characterization measurements must resolve this spectral structure at a level of precision consistent with the performance goals for the redeveloped lunar reference.

It is well known that the spectral content of moonlight varies with phase, with the Moon becoming redder as the phase angle increases [15]. Different lunar terrains (e.g., maria and highlands) vary in their albedo, spectral signatures and reflectance phase functions, leading to spectral dependencies in the lunar irradiance for different phase angles and librations caused by sampling different distributions of terrains. This behavior can only be sensed with spectrally resolved measurements. Its magnitude has not yet been determined definitively, as existing lunar irradiance datasets lack the spectral precision needed to conduct the necessary analyses reliably. To develop a capability to predict these effects requires collecting Moon characterization measurements that capture the spectral content of the lunar irradiance over the full geometric ranges of phase angle and librations. In practice, an advanced lunar model may need to query a database of spectral signatures corresponding to the observed distributions of lunar terrains.

\subsection{Polarization Specification}

The polarized nature of moonlight will affect instruments' lunar measurements in proportion to their polarization sensitivity. Spatially integrated moonlight is polarized up to $\sim 13 \%$ dependent on wavelength, and varying widely with phase angle, passing through zero at about $24^{\circ}$ phase [16]. The degree of polarization also varies with lunar terrain type, leading to a small libration dependence. As moonlight polarization is an inherent property of the Moon, the capability to predict it must be a component of a lunar reference; however, the instrument-dependent nature of its impact means this capability likely will need to be developed and utilized separately from the radiometric reference.

To develop a lunar polarization model that is complementary to an irradiance reference requires a database of full-disk polarization measurements spanning the same range of phase angles and librations that are sampled for radiometry. No such measurements are known to have been collected since the 1960s. Characterizing the wavelength dependence requires some spectrally resolved measurements, to verify existing empirical formulations [16] or to make refinements to them.

\section{Example Projects to Acquire New Lunar Irradiance Measurements}

The desire to utilize an advanced lunar calibration capability has prompted movements to improve the lunar reference, and it is recognized that a prerequisite step is to collect new, high-accuracy Moon characterization measurements. A number of independent projects to acquire lunar measurements have been initiated by institutions around the world, and these are addressing requirements for long-term, spectrally resolved observations, and are pushing the limits of absolute radiometric accuracy. We present here three examples of currently active NASA-sponsored projects that have these objectives.

\subsection{Air-LUSI}

The airborne Lunar Spectral Irradiance (air-LUSI) project has the goal to acquire dedicated lunar irradiance measurements with sub-percent absolute accuracy from an aircraft platform flying at 70,000 feet $(21.6 \mathrm{~km})$ altitude. The project is a joint effort of NASA, the National Institute of Standards and Technology (NIST), USGS, the University of Guelph (Canada), and Hawk Institute for Space Science. The non-imaging air-LUSI irradiance sensor views the Moon from the wing pod of the NASA ER-2 high-altitude aircraft. As of this writing, air-LUSI has successfully completed a 5-night demonstration campaign that sampled phase angles of $9.4^{\circ}, 21.0^{\circ}, 33.3^{\circ}, 45.9^{\circ}$ and $58.6^{\circ}$, all after Full Moon. 
The schematic diagram in Figure 3 shows the basic instrument layout. The telescope consists of an objective lens with a small integrating sphere at its focus, which spatially integrates the radiance within the telescope field of view. Light from the sphere is conducted through a fiber optic cable to a field spectrometer located in an environmentally controlled segment of the ER-2 wing pod. This instrument is a Czerny-Turner design grating spectrograph with a back-thinned CCD focal plane array, covering a spectral range approximately 300 to $1100 \mathrm{~nm}$, with $3.7 \mathrm{~nm}$ spectral resolution and $0.8 \mathrm{~nm}$ spectral sampling across the entire range. Critical optical components are thermally controlled to maintain radiometric stability, and a LED light source can illuminate the integrating sphere for validation of the spectrometer responsivity during aircraft ascent and descent. A dry nitrogen purge prevents condensation and/or icing during descent.

\section{SUBSYSTEM DIAGRAM}

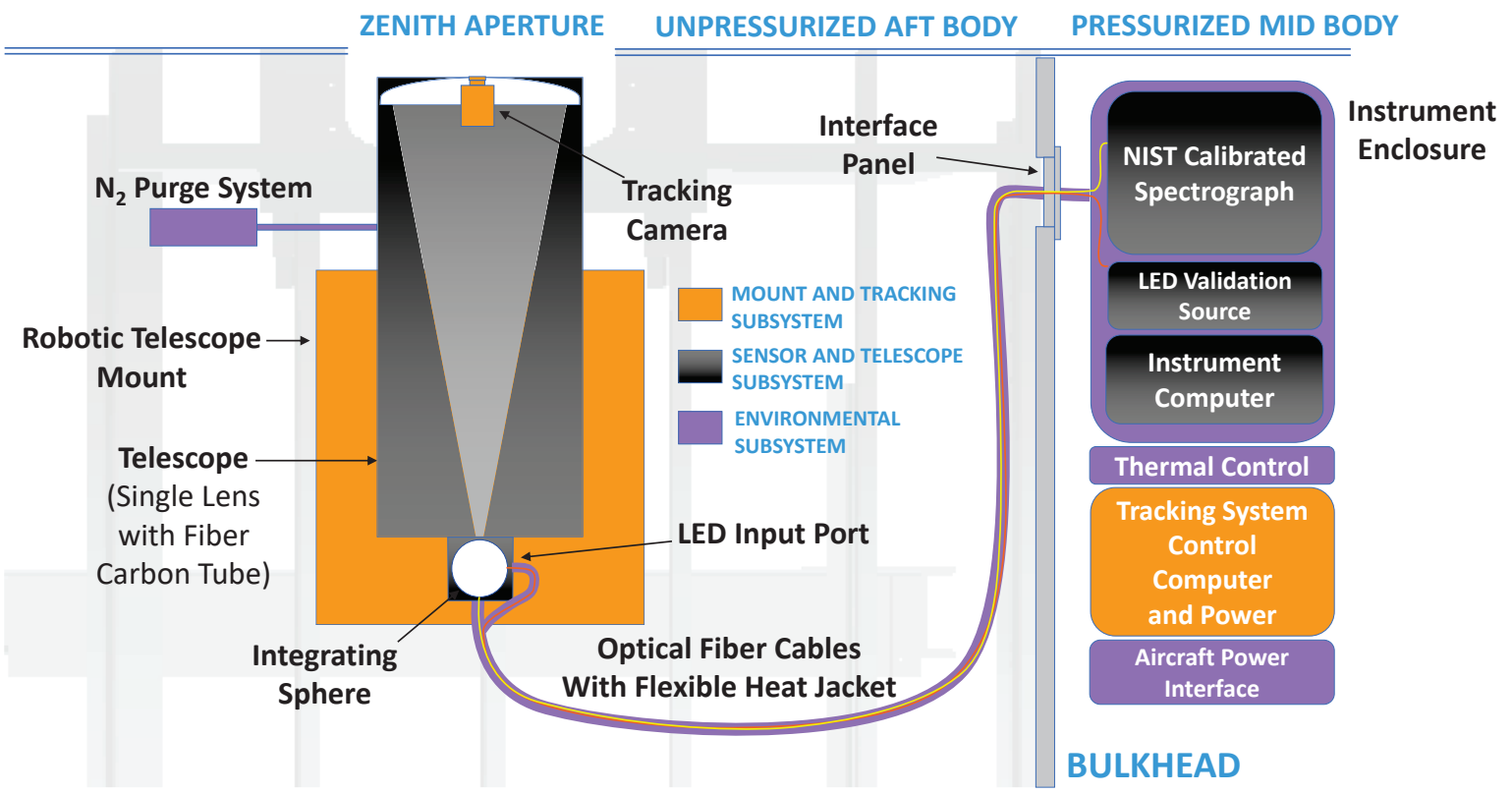

Figure 3. Schematic diagram of air-LUSI instrument components and their placement in the ER-2 wing pod.

The telescope is mounted in a 2-axis pointing gimbal that autonomously tracks the Moon, compensating for the normal motions of the aircraft during flight. A framing camera attached to the telescope tube provides Moon images to a robotic feedback loop that controls the gimbal actuators and keeps the Moon disk at a specified position in the camera frame, which is boresighted to the telescope field of view. Under flight conditions, this system kept the Moon at its targeted position within two tracking camera pixels $\left(0.10^{\circ}\right)$ more than $95 \%$ of the collection time.

Ground calibrations are performed by pointing the air-LUSI instrument to an integrating sphere source positioned at a distance so that its exit port subtends a solid angle similar to the Moon's size. The source also is measured with a second field spectrometer operating as a transfer radiometer, whose calibration is traced to NIST primary optical power standards. These calibrations are done in a rigorous sequence that is repeated several times at NIST before and after each flight campaign, and again at the airfield before the instrument system is uploaded onto the aircraft and after it is removed from the aircraft. Additional calibrations are done with the instrument installed in the wing pod immediately after upload, just before offload, and between each of the flights. This protocol is expected to achieve absolute calibration uncertainty under $0.5 \%(\mathrm{k}=1)$ for visible wavelengths.

Flights are conducted out of NASA's Armstrong Flight Research Center in Palmdale, California. After ascent to nominal operating altitude $(\sim 70,000$ feet), the flight path follows a heading perpendicular to the direction toward the Moon, allowing air-LUSI to view it through a rectangular 
transverse slot in the top of the wing pod. The acquisition sequence begins by slewing the telescope from its stowed position to the expected location of the Moon in the sky, then the tracking system centers the disk on the boresight position. Irradiance data are collected in triplets of $5 \mathrm{~s}$ integration time over a total collection time of about $30 \mathrm{~min}$. The telescope is then stowed, the $\mathrm{N}_{2}$ purge system is activated, and the aircraft descends for landing about two hours after takeoff.

\subsection{ARCSTONE}

ARCSTONE (not an acronym) is a small-satellite mission dedicated to acquiring high-accuracy lunar spectral reflectance measurements from outside the atmosphere, with sufficient mission duration and density of observations to develop an SI-traceable lunar irradiance reference. Details of the ARCSTONE design, development and concept of operations are found in [17]. The measurement concept captures the whole disk of the Moon or Sun with no change of optical path or elements, using the solar measurements to calibrate the lunar observations to reflectance. The measurement uncertainty goal is $<0.5 \%(\mathrm{k}=1)$ for spectral reflectance. SI-traceable solar spectral irradiance (SSI) values concurrent with the ARCSTONE acquisitions will be used to convert the measured reflectances into SI-traceable lunar irradiances.

The ARCSTONE spectrometer has a compact design sized to fit in the payload space of a 6U-form CubeSat. Figure 4 shows the layout of the instrument and its major components: optical bench, flexure mounts, mercury-cadmium-telluride (MCT) detector, detector cryocooler, cryocooler and control electronics; and the instrument packaging into a $6 \mathrm{U}$ CubeSat bus. The remaining payload space holds the spacecraft control and instrument interface components. The ARCSTONE instrument concept includes a slit-limited beam expander as front optics, grating, focusing optics, and an array detector. This design provides the ability to measure both the Sun and the Moon with the same optical path using a technique that accommodates the solar/lunar intensity difference solely by varying the detector integration times, which are controllable at the nanosecond level, while also maintaining sufficient signal-to-noise ratios at lunar light levels. Wavelength coverage spans the range $350 \mathrm{~nm}$ to $2300 \mathrm{~nm}$ with $4 \mathrm{~nm}$ to $6 \mathrm{~nm}$ spectral sampling.

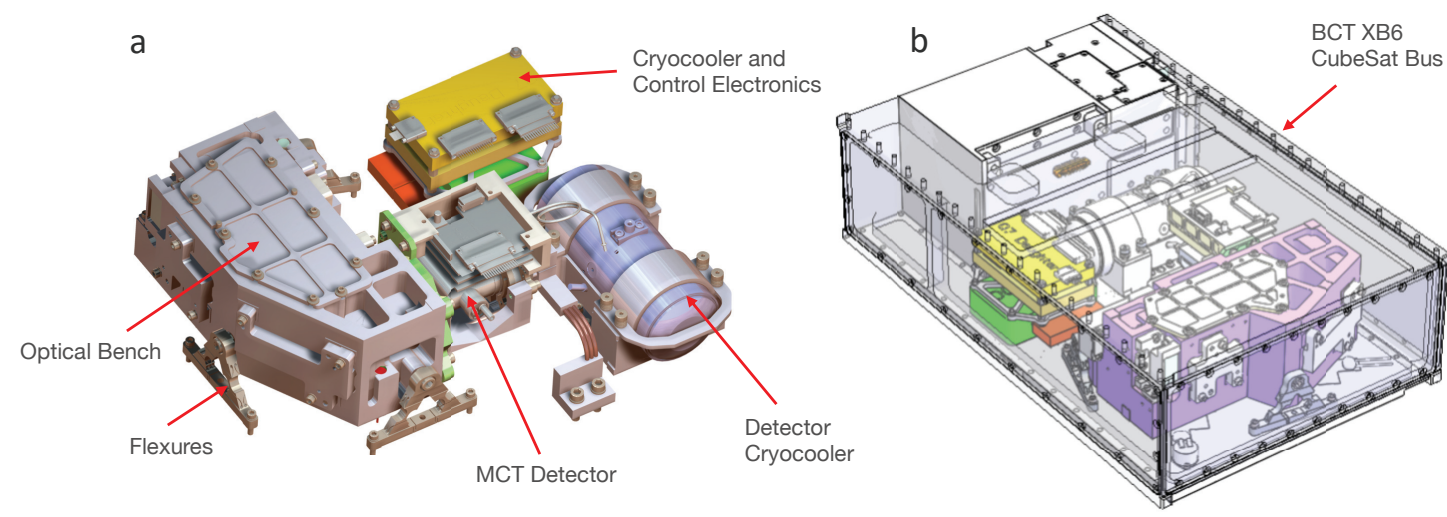

Figure 4. Layout of the ARCSTONE sensor components (a) and the instrument packaging into a 6U CubeSat bus (b).

ARCSTONE will observe the Moon from a Sun-synchronous or polar orbit every $12 \mathrm{~h}$, where this cadence was selected for sufficient sampling of different observation geometries while respecting data downlink considerations. Phase angles up to $135^{\circ}$ will be sampled, expanding the capability for on-orbit lunar calibration beyond the current 90-degree limit to about three weeks per month. Lunar acquisitions will occur at the highest latitudes of the orbit, to sample the largest range of lunar latitude librations. Each lunar measurement sequence will last approximately $5 \mathrm{~min}$, and consist of multiple acquisitions of $\sim 15 \mathrm{~s}$ integration time. This sampling time constraint is imposed by the ARCSTONE measurement accuracy objectives-from low Earth orbit, the lunar irradiance varies up to $0.1 \%$ in 
$16 \mathrm{~s}$ due to changes in both the Moon-sensor distance and the phase angle caused by the platform motion. Solar measurements will be acquired at least weekly. Pointing to the Moon and Sun will be accomplished using spacecraft maneuvers, as the spectrometer will be fixed to the satellite structure.

The cadence and duration of ARCSTONE lunar acquisitions are intended to provide sufficient phase angle and libration coverages to meet requirements for constructing a lunar irradiance reference (i.e., a model). To fill out the libration parameter space requires at least 3 years of continuous on-orbit operation, depending on the starting date.

\subsection{CLARREO Pathfinder}

The Climate Absolute Radiance and Refractivity Observatory (CLARREO) mission is proceeding with a Pathfinder project (https: / / clarreo-pathfinder.larc.nasa.gov) having objectives to demonstrate technologies required for: (1) Earth reflectance measurements with unprecedented absolute accuracy, $\leq 0.3 \% \quad(\mathrm{k}=1)$, and (2) reference inter-calibration of other Earth observing sensors in orbit. The CLARREO Pathfinder (CPF) instrument is a reflected solar-wavelength imaging spectrometer based on the HyperSpectral Imager for Climate Science (HySICS, [18]). The swath width is $10^{\circ}$ with $0.1^{\circ}$ spatial resolution, and spectral coverage is $350 \mathrm{~nm}$ to $2300 \mathrm{~nm}$ with $\sim 3 \mathrm{~nm}$ sampling across the entire wavelength range. The CPF instrument will be deployed on the International Space Station (ISS) at Express Logistics Carrier 1 (ELC-1) Site 3, as shown in Figure 5. Nominal operations will be mostly nadir viewing with spectral imaging in pushbroom mode, plus off-nadir Earth scene acquisitions for inter-calibration with Clouds and Earth's Radiant Energy System (CERES) and Visible Infrared Imaging Radiometer Suite (VIIRS) sensors. Views of the Moon are planned as observations of opportunity.

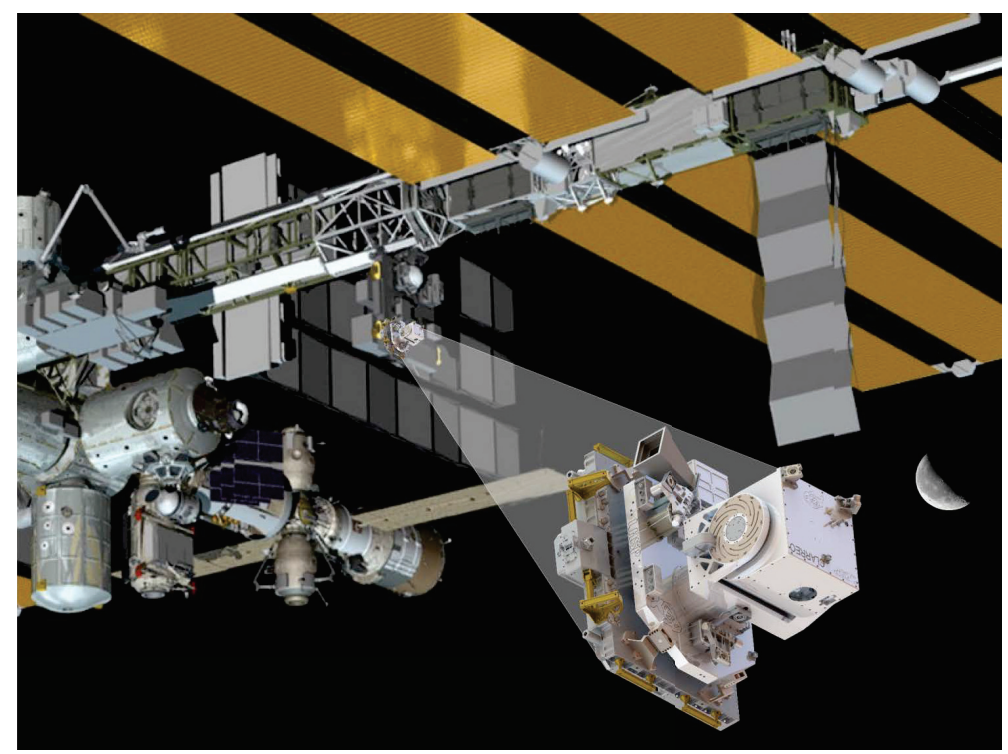

Figure 5. Concept diagram of the CLARREO Pathfinder instrument and its installation location on the International Space Station at Express Logistics Carrier 1, Site 3.

To enable off-nadir views for reference inter-calibration, the CPF spectrometer is mounted in a 2-axis gimbal. The roll-over-pitch gimbal configuration induces an effect where the slit footprint rotates on the target as an across-slit scan progresses. Consequently, lunar images will be differentially oversampled, with the oversampling dependent on the spatial position in spectral image frames. To determine the actual oversampling of the Moon disk for these scans will require precise knowledge of the sensor pointing (in an inertial reference frame) and the gimbal positions and slew rates, as well as the usual sample timing and detector spatial response functions. The spatial analysis techniques developed to produce lunar irradiances from these data, with the goal of absolute measurement accuracy approaching the CPF sensor calibration uncertainty, will represent a significant technological advancement in the processing of spectral images acquired by space-based instruments. 
This technology development is likely to transfer to irradiance measurements from any line-scanned images of the Moon.

Although the CPF field of regard will be constrained to views toward the port side of the ISS, studies of possible clear lines of sight to the Moon suggest there will be numerous opportunities for lunar observations during the nominal one year of flight operations. Figure 6 shows the lunar phase and libration coverages determined from a simulation of potential CPF Moon observations having $4 \mathrm{~min}$ or longer duration. Although transitory obstructions by the ISS solar panels are expected to impact the actual number of Moon viewing opportunities available, the potential coverage of observation geometries is substantial. The CPF instrument will acquire imaging data of the Moon which can be integrated over entire lunar disk and combined with its solar irradiance calibration to provide lunar spectral reflectance measurements.

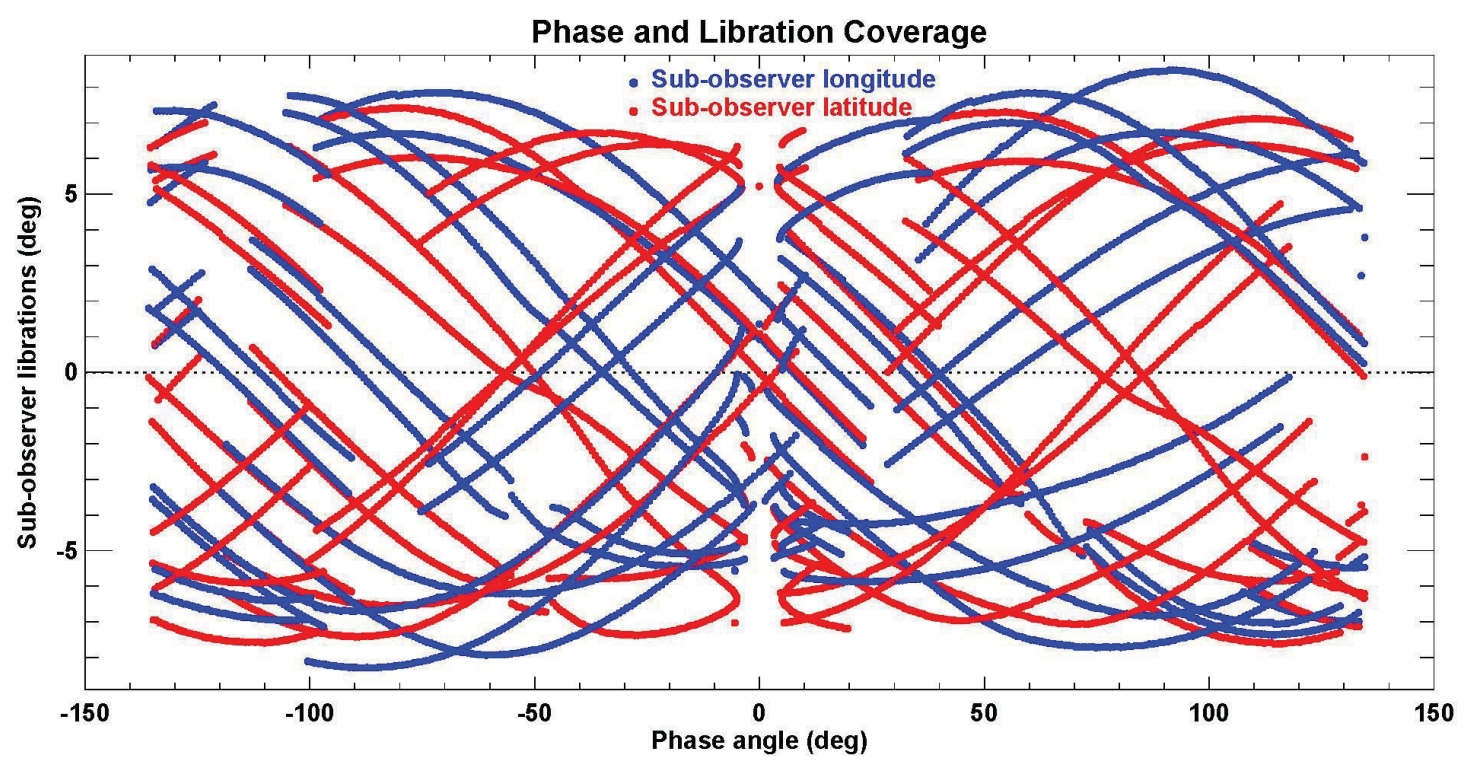

Figure 6. Results of a simulation of Moon viewing opportunities by CLARREO Pathfinder from the ISS ELC-1 Site 3. Potential observations of $4 \mathrm{~min}$ or longer duration are represented as colored dots.

\section{Revising the Lunar Reference Using New Measurements}

The lunar measurement activities described in the preceding section represent examples of recent efforts to supplement our current quantitative knowledge of the absolute lunar spectral irradiance. Other Moon observation projects are in development at various institutions worldwide, and these systems are expected to come online in the near future. A common, high-priority objective is verifiable radiometric accuracy with quantified uncertainty evaluations.

Eventually, newly acquired Moon characterization datasets may be adequate to support lunar modeling on their own, but the length of time needed to acquire a viable set of measurements is substantial, whereas the need for improved accuracy of the lunar reference is immediate. An example of this urgency is the application of nighttime aerosol properties retrievals, for which the Moon is the exo-atmospheric light source used for photometry [8]. Here we present conceptual short-term and long-term tasks that can utilize new, high-accuracy lunar measurements to improve and eventually redevelop the model-based lunar spectral irradiance reference.

\subsection{Improvements to the Current Lunar Model}

Initially, new Moon characterization datasets can be presumed to have limited phase angle and libration coverages. But a sparse set of measurements can serve as reference points for the existing lunar model, allowing to constrain the absolute scale and the spectral specification of the irradiance. With sufficient sampling, they also can constrain the model's phase angle specification. 
As explained in Section 2.2, the ROLO model post-processing procedure fits a reference lunar reflectance spectrum to the 32 reflectance outputs from Equation (4), and the irradiance is derived from this fitted spectrum. The reference spectrum was developed from combining laboratory reflectance measurements of returned Apollo samples, where the proportions of the different sample spectra were determined from a best fit to the spectral signatures exhibited by the ROLO data. This was found to also mimic the spectral content of other full-disk Moon observations.

A new set of spectrally resolved lunar irradiance measurements has the potential to better define the spectral shape of the full-disk lunar reflectance, and also its phase angle dependence. New irradiance spectra, converted to reflectance, can be used to select and combine laboratory spectra with more robust constraints than the original ROLO development, leading to a more accurate reference reflectance spectrum for use with the model post-processing routines. This procedure can be applied to a series of observed phase angles, providing an improved evaluation of the lunar phase reddening effect and quantifying the variations in spectral content with phase.

Once a revised reference reflectance spectrum has been produced and installed in the lunar model post-processing system, absolute spectral irradiances can be compared between the new high-accuracy measurements and their corresponding model-generated irradiance spectra. Since these individual comparisons are for specific phase angles and librations, any absolute differences seen do not necessarily reflect synoptic scale offsets. However, the existing lunar model can be used to generate adjustments that map these as-observed conditions into specified representative geometries, which collectively can produce more generally applicable absolute scale corrections. Additionally, a phase angle series of new measurements could reveal a systematic phase-dependent bias in the model, if one exists. In that case, it may be possible to produce spectrally resolved phase corrections after applying adjustments to correct for the libration effects.

\subsection{Redeveloping the Lunar Reference Incorporating New Measurements}

To transfer the improved accuracy of new lunar measurements to an operational radiometric reference will require revising the model specification of how the lunar irradiance varies with observation geometry (phase angle and librations) and wavelength. Ongoing efforts to acquire high-accuracy lunar measurements eventually will provide a reasonably complete picture of the Moon's brightness variations as observed from the ground or low Earth orbit, potentially adequate to use as a basis for modeling on its own. However, the ROLO collection of more than 110,000 lunar images retains a potent utility for characterizing the Moon's radiometric behavior, providing a strong incentive for constructing a combined dataset that includes both the ROLO archive and the new, high-accuracy, spectrally resolved measurements. This can be accomplished regardless of the extent of observation geometries covered by the new measurements.

A pragmatic approach to constructing a new lunar irradiance model can consider the model as composed of two parts, both having dependence on wavelength $\lambda$ : an absolute spectral irradiance $E_{r}(\lambda)$ at a reference geometry (e.g., a fixed phase angle and zero sub-observer librations), and a dimensionless irradiance variation factor $\mathcal{F}(P, \lambda)$, where $P$ captures all the photometric angles (signed phase angle and sub-solar and sub-observer librations) collectively. Then: $E(P, \lambda)=E_{r} \cdot \mathcal{F}$.

The accuracy of such a model depends upon both of these terms; however, they may be determined somewhat independently. $E_{r}(\lambda)$ requires high-accuracy measurements with moderate spectral resolution, and which are not impacted by the Earth's atmosphere. $\mathcal{F}(P, \lambda)$ can be developed using the existing large set of lunar observations, after scaling each instrument's measurements to a common irradiance specification, which is an iterative process.

Combining the available lunar datasets will require some form of pre-processing to conform all the measurements to a common spectral scale. This spectral pre-processing is facilitated by the lunar reflectance spectrum being smooth, nearly featureless, and not highly sloped. A weighting scheme also must be implemented that reflects the relative uncertainties of the measurements. Because many 
lunar observations are concentrated around particular phase angles, techniques with considerable complexity may be required to create a unified characterization dataset for modeling.

These redevelopment efforts will create a new analytic model formulation to describe the variations in lunar disk reflectance with geometry, $\mathcal{F}(P, \lambda)$. The current successful representation of the Moon's photometric behavior by the ROLO model suggests that some similarity to its form should be retained, particularly for the terms that describe the primary phase dependence, the waxing/waning difference and the opposition effect. Revision of the libration terms may include the sub-solar latitude. Approaches currently under study include normalizing the existing lunar observations to $E_{r}(\lambda)$ and specifying the geometric terms similar to those in Equation (4) as polynomials in wavelength, providing a $\mathcal{F}(P, \lambda)$ that is continuous in both $\lambda$ and all the photometric angles $P$.

Testing a new lunar model formulation can be conducted using archives of measurements acquired by spacecraft instruments, and the high-accuracy lunar measurements themselves. The Moon's photometric invariance means an improved lunar model can predict the brightness for any time the Moon was observed; therefore, a metric of the new model's success would be a reduction in the mean absolute residuals of comparisons between past measurements from space-based sensors and the corresponding new model outputs. The geometric variation function $\mathcal{F}(P, \lambda)$ also can be used to generate factors that map the high-accuracy measurements into identical observation geometries. These adjusted measurements should coincide within the combined uncertainty of the measurements and the model, regardless of the individual observation geometries.

\section{Conclusions}

The inherent photometric stability of the Moon has been leveraged to develop a radiometric reference from moonlight at reflected solar wavelengths, and the existing lunar calibration system has successfully met critical on-orbit calibration needs such as for ocean color measurements. Improving the accuracy of the lunar reference can unlock the potential for additional calibration capabilities that have high value for space-based climate measurements, including providing a stable, common reference for sensor inter-calibration and enabling SI-traceable absolute calibration against the Moon directly. To achieve these milestones requires at least an order of magnitude accuracy improvement over current lunar photometric models.

An absolute lunar reference with order of tenths-percent uncertainty is technically feasible, but this requires collecting new Moon characterization measurements with compatible levels of accuracy, with traceability to absolute radiometric standards, and with sufficient phase angle and libration coverages to support precise lunar modeling. Spectrally resolved measurements are necessary to capture variations in the Moon's spectral content for different observing geometries. A complete lunar characterization dataset also should include polarization measurements, to be used for development of a separate and supplemental polarization model that permits accurate lunar calibration for polarization-sensitive sensors. Refinement and eventually redevelopment of lunar models will be required to capture and reflect the higher accuracy of new Moon characterization measurements.

The availability of a high-accuracy lunar reference can be expected to motivate renewed efforts to refine the data processing techniques used to derive irradiance measurements from calibration observations of the Moon. Comparisons of measurements made by spacecraft instruments to their corresponding lunar reference values often show disagreements that exceed the combined uncertainty of the current reference and the sensors' radiometric calibrations. A high-accuracy lunar reference will place emphasis on the instruments' lunar measurement uncertainties. A long-term objective is a substantial increase in the precision of lunar measurements used for radiometric calibration, to parallel ongoing advances in lunar model development.

Author Contributions: Lunar calibration project (ROLO) operations, algorithm and software development, model validation, data curation, funding acquisition and project administration, T.C.S.; original ROLO facility development and advanced lunar modeling, H.K.; ARCSTONE and CLARREO Pathfinder, C.L.; air-LUSI, K.T. All authors have read and agreed to the published version of the manuscript. 
Funding: Original funding for establishing and operating the ROLO facility and developing the lunar irradiance model was provided by the NASA Earth Observing System (EOS) program. Ongoing support for the ROLO project has been provided by the USGS Land Satellites Data System (LSDS) program through the Earth Resources Observation and Science (EROS) Calibration/validation Center Of Excellence (ECCOE). Support for manuscript preparation was provided by NASA Langley Research Center through the CLARREO Pathfinder project under Interagency Agreement NNL20OB04A.

Conflicts of Interest: The authors declare no conflict of interest.

\section{References}

1. Kieffer, H.H. Photometric Stability of the Lunar Surface. Icarus 1997, 130, 323-327. [CrossRef]

2. Eplee, R.E., Jr.; Meister, G.; Patt, F.S.; Barnes, R.A.; Bailey, S.W.; Franz, B.A.; McClain, C.R. On-orbit calibration of SeaWiFS. Appl. Opt. 2012, 51, 8702-8730. [CrossRef] [PubMed]

3. Kieffer, H.H.; Stone, T.C. The spectral irradiance of the Moon. Astronom. J. 2005, 129, 2887-2901. [CrossRef]

4. Anderson, J.M.; Becker, K.J.; Kieffer, H.H.; Dodd, D.N. Real-Time Control of the Robotic Lunar Observatory Telescope. Pub. Astron. Soc. Pac. 1999, 111, 737-749. [CrossRef]

5. Hayes, D.S. Stellar absolute fluxes and energy distributions from 0.32 to 4.0 microns. In Calibration of Fundamental Stellar Quantities, Proceedings of the IAU Symposium No. 111, Como, Italy, 24-29 May 1984; D. Reidel Publishing Co.: Dordrecht, The Netherlands, 1985; pp. 225-252.

6. Strecker, D.W.; Erickson, E.F.; Witteborn, F.C. Airborne stellar spectrometry from 1.2 to 5.5 microns-Absolute calibration and spectra of stars earlier than M3. Astrophys. J. Suppl. 1979, 41, 501-512. [CrossRef]

7. Castelli, F.; Kurucz, R.L. Model atmospheres for Vega. Astron. Astrophys. 1994, 281, 817-832.

8. Barreto, A.; Román, R.; Cuevas, E.; Pérez-Ramirez, D.; Berjón, A.J.; Kouremeti, N.; Kazadzis, S.; Groebner, J.; Mazzola, M.; Toledano, C.; et al. Evaluation of night-time aerosols measurements and lunar irradiance models in the frame of the first multi-instrument nocturnal intercomparison campaign. Atmos. Environ. 2019, 202, 190-211. [CrossRef]

9. Thuillier, G.; Hersé, M.; Labs, D.; Foujols, T.; Peetermans, W.; Gillotay, D.; Simon, P.C.; Mandel, H. The Solar Spectral Irradiance from 200 to $2400 \mathrm{~nm}$ as Measured by the SOLSPEC Spectrometer from the ATLAS and EURECA Missions. Sol. Phys. 2003, 214, 1-22. [CrossRef]

10. Wehrli, C. Extraterrestrial Solar Spectrum; Physikalisch-Meterologisches Observatorium Davos and World Radiation Center: Davos-Dorf, Switzerland, 1985.

11. Pieters, C.M.; Mustard, J.F. Exploration of crustal/mantle material for the Earth and Moon using reflectance spectroscopy. Rem. Sens. Environ. 1988, 24, 151-178. [CrossRef]

12. Wilson, T.; Angal, A.; Xiong, X. Sensor performance assessment for Terra and Aqua MODIS using unscheduled lunar observations. Proc. SPIE 2018, 10785, 1078519.

13. Sun, J.; Xiong, X.; Barnes, W.L.; Guenther, B. MODIS Reflective Solar Bands On-Orbit Lunar Calibration. IEEE Trans. Geosci. Remote Sens. 2007, 45, 2383-2393. [CrossRef]

14. Xiong, X.; Sun, J.; Fulbright, J.; Wang, Z.; Butler, J.J. Lunar Calibration and Performance for S-NPP VIIRS Reflective Solar Bands. IEEE Trans. Geosci. Remote Sens. 2016, 54, 1052-1061. [CrossRef]

15. Lane, A.P.; Irvine, W.M. Monochromatic phase curves and albedos for the lunar disk. Astronom. J. 1973, 78, 267-277. [CrossRef]

16. Dollfus, A.; Bowell, E. Polarimetric Properties of the Lunar Surface and its Interpretation. Part I. Telescopic Observations. Astron. Astrophys. 1971, 10, 29-53.

17. Swanson, R.; Kehoe, M.; Stebbins, M.; Courrier, H.; Lukashin, C.; Jackson, T.; Cooney, M.; Davis, W.; Young, C.; Kopp, G.; et al. The ARCSTONE Project to Calibrate Lunar Reflectance. In Proceedings of the 2020 IEEE Aerospace Conference, Big Sky, MT, USA, 7-14 March 2020.

18. Kopp, G.; Smith, P.; Belting, C.; Castleman, Z.; Drake, G.; Espejo, J.; Heuerman, K.; Lanzi, J.; Stuchlik, D. Radiometric flight results from the HyperSpectral Imager for Climate Science (HySICS). Geosci. Instrum. Method. Data Syst. 2017, 6, 169-191. [CrossRef]

(C) 2020 by the authors. Licensee MDPI, Basel, Switzerland. This article is an open access article distributed under the terms and conditions of the Creative Commons Attribution (CC BY) license (http:/ / creativecommons.org/licenses/by/4.0/). 